\title{
Design of OBDH Subsystem for Remote Sensing Satellite based on Onboard Route Architecture
}

\author{
WANG Wenping, WANG Xianghui, HAN Bo, ZHANG Yahang, and LEI Yong \\ Beijing Institute of Spacecraft System Engineering, Beijing 100094, China
}

\begin{abstract}
This paper describes the On-Board Data Handling Subsystem of remote sensing satellite, which plays nowadays a relevant role to support satellite assembling, integration and testing activities in the implementing complex system-level requirements. Firstly, the architecture and functionalities of On-Board Data Handling Subsystem are described. Secondly, layered On-Board Data Handling Subsystem flight software architecture based on onboard route as well as software maintenance and tolerance designs are depicted. In particular, we present the redundancies and fault processing means for ensuring the correctness of On-Board Data Handling Subsystem.
\end{abstract}

\section{Introduction}

In this paper, we present the architectural approach of the On-Board Data Handling Subsystem (OBDH S/S) running on the China-Brazil Earth Resource Satellite onboard computer. OBDH S/S is one of the subsystems, which belongs to the service platform of remote sensing satellite. Satellite $\mathrm{OBDH} \mathrm{S} / \mathrm{S}$ can be regarded as a system-level entity, which plays a relevant role in implementing some complex requirements, and is the main interface for the ground operators. OBDH $\mathrm{S} / \mathrm{S}$ integrates the TT\&C video frequency functions into a system based on microprocessor equipment to perform the telecommand(TC), telemetry(TM), program control, auto-control onboard, housekeeping functions for the satellite and generate on-board time reference, etc. The $\mathrm{OBDH} \mathrm{S} / \mathrm{S}$ life cycle and reliability requirements are to be defined at the very beginning of any satellite project [1].

Satellites are becoming increasingly softwaredependent, together with other complex systems [2]. The $\mathrm{OBDH}$ flight software (OBDH F/S) provides all the necessary functions for nominal and emergency operations of the satellite and enables the ground operators to control and supervise the various the state of all sub-systems for the whole satellite mission.

This paper is organized as follows. Section 2 describes its layered architecture. Section 3 outlines the OBDH S/S functionality. Section 4 presents the flight software components based architecture as well as some software maintenance and tolerance designs. Section 5 focuses on the redundancies and fault processing means to fulfill the need of having a robust, reusable, modular OBDH S/S. Finally, section 6 concludes the paper.

\section{Architecture descriptions}

The deployment architecture diagram of OBDH S/S running on the on-board computer is shown in Fig.1. The OBDH S/S shall be configured with two-level distribution structure by means of a data bus with the core of the fault tolerant computer. It comprises the following units: a Central Terminal Unit(CTU), several Remote Terminal Units(RTUs), a Telecommand Unit(TCU), a Command Decode Unit(CDU), a UltraStable Oscillator(USO) and a Data Recording and Processing unit(DRP). Flight software are mounted on the different intelligent units.

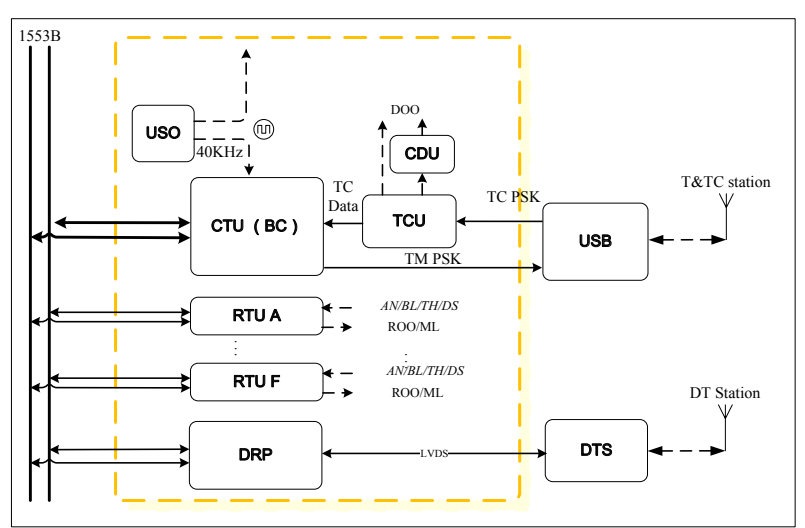

Fig. 1. OBDH Subsystem topology

\subsection{Telecommand unit-TCU}

The TCU of OBDH S/S is an internal hot redundant unit, which performs the functions of receiving the TC video signal from S-band receiver, demodulating synchronization of the TC signal, decoding and validating the TC frame(including the Address and Synchronization Word (ASW), mode and message codes), distributing and executing the direct ON/OFF command to the satellite subsystem, sending the TC

Corresponding author: wangwenping304@163.com 
message code to the CDU or CTU through the Serial Load Command (SLC) interface according to the mode, and controlling the data injection channels configuration.

\subsection{Command decode unit-CDU}

The CDU of OBDH S/S is also an internal hot redundant unit, which has the ability of receiving a serial message from the TCU and generating the corresponding direct ON/OFF command to the users.

\subsection{Central terminal unit-CTU}

The CTU is the core unit of OBDH S/S, which shall be cold redundancy in one box and shall perform the following functions.

The CTU is the central terminal unit of satellite, which plays the relevant role to process and distribute the routed commands \& data and on-board commands. Routed commands and data are received from the ground station and on-board commands are generated by onboard computer based on some parameter measurements and a control algorithm. The types of the routed commands and data include real-time/time-tagged ON/OFF commands, real-time/time-tagged Memory Load Commands (MLC), OBDH housekeeping data, Attitude and Orbit Control Sub-system (AOCS) control data and payload control data, etc. In addition, the CTU performs the functions of executing program-control commands, command group and time-tagged commands, and deleting time-tagged commands (groups).

\subsection{Remote terminal unit-RTU}

The OBDH S/S owns several RTUs, which are identical and are distributed among the different positions in the satellite to provide the direct $\mathrm{TM} / \mathrm{TC}$ interfaces to the satellite subsystems. Each RTU is identified by the SDB remote terminal address. The microprocessor-based RTU provides the ability of TM acquisition, command distribution and some data processing. The operation of the RTU is controlled by the CTU via the SDB communication.

The RTU provides four kinds of channels for the acquisition of telemetry data: analog channels (AN), digital bi-level channels (BL), serial digital channels (DS) and thermal channels (TH). An analog channel is sampled by the RTU and then converted into an 8 bit binary word. A digital bi-level channel is two-level digital information delivered on a single line. A serial digital word is transferred from user to the RTU with the control of a sampling signal and an acquisition clock signal. A thermal channel is the same as the analog channel.

There are two kinds of interface channels to distribute and execute of commands and data to users: ON/OFF command channels and Memory Load Command (MLC) channels. The RTU also provide the function of self-testing, which includes the tests of CPU,
RAM, PROM, AN, DS, ON/OFF command channel and MLC channel.

\subsection{Ultra-stable oscillator-USO}

Ultra-Stable Oscillator is an internal cold redundant unit outputting high-stability $40 \mathrm{kHz}$ and $5 \mathrm{MHz}$ clock; $40 \mathrm{KHz}$ clock to CTU, which is utilized to generate the on-board time reference and $5 \mathrm{MHz}$ clock to GPS.

\subsection{Data recording and processing unit-DRP}

Data recording and processing unit is an internal cold redundant unit, which receives satellite $\mathrm{TM}$ or housekeeping data from CTU through SDB bus and playbacks the data from TM downlink channel (on-orbit inquire mode)and Data Travelling Subsystem channel(DTS playback mode). DRP has the ability of saving the important data of OBDH, AOCS, and etc.

In On-orbit inquire mode, the ground controller can downlink eight frames of specially selected TM data packets each time, according to the packet APID, or the packet saved time scale uploaded.

In DTS playback mode, the ground controller can upload the time-tagged command to playback all the TM data from DTS.

\section{Functional requirements}

In order to support the platform and payload operations, the OBDH S/S shall provide the following functions.

For the TC routing, execution and report generation, the $\mathrm{OBDH} \mathrm{S} / \mathrm{S}$ should be able to receive, demodulate and decode the telecommand messages from ground and distribute telecommands to all the satellite subsystems (see Fig.2). Usually, it shall handle three types of commands, the direct ON/OFF commands, routed commands and uplink data injection. In addition, the capability of performing program-control commands, on-board commands, command groups and time-tagged commands should be provided.

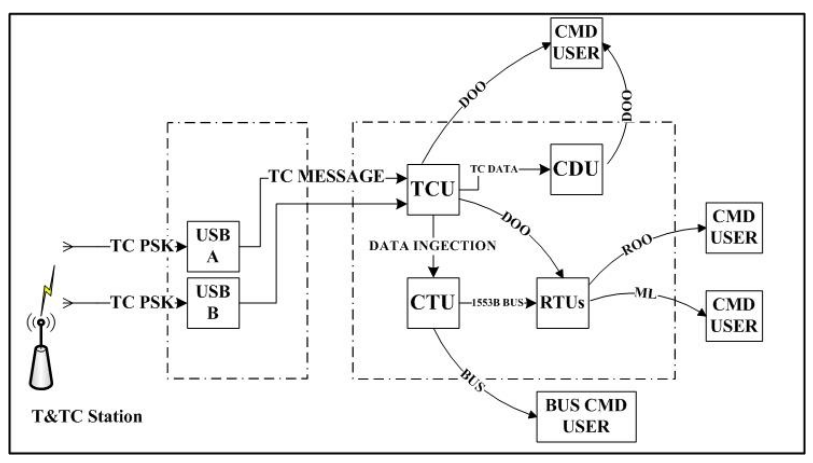

Fig. 2. Satellite telecommand receiving, routing and execution

In order to report relevant satellite parameters values and on-board operational status, the OBDH S/S regularly gathers and encodes the satellite telemetry data, and sends TM packets to the ground in real-time via S-band telemetry transmitter or stored in the on-board memory 
for later down-link (see Fig.3). The CTU generates the OBDH S/S important data, and stores or restores the important data between the OBDH S/S(CTU, RTU, DRP) and AOCS. The CTU also provides the ability of dumping all the time-tagged command queues (On/Off, MLC and SDB) by using one single telecommand from the ground station.

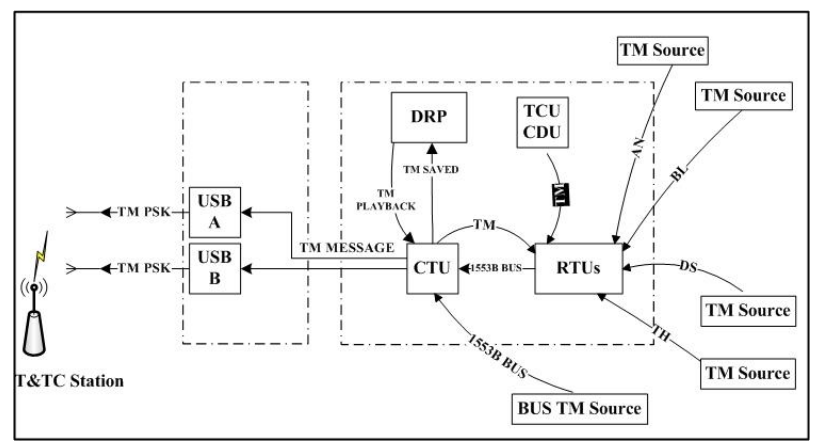

Fig. 3. Satellite telemetry data gathering, encoding and downlink

Generally, all the satellite data, which come from RTUs, AOCS, GPS receiver, thermal controller etc, are gathered and formatted into Advanced Orbiting System (AOS) frame [3-5]. Fig.4 describes how the $\mathrm{OBDH}$ handles the TM data based on the AOS. In addition, the capability of changing telemetry scheduling period should be provided.

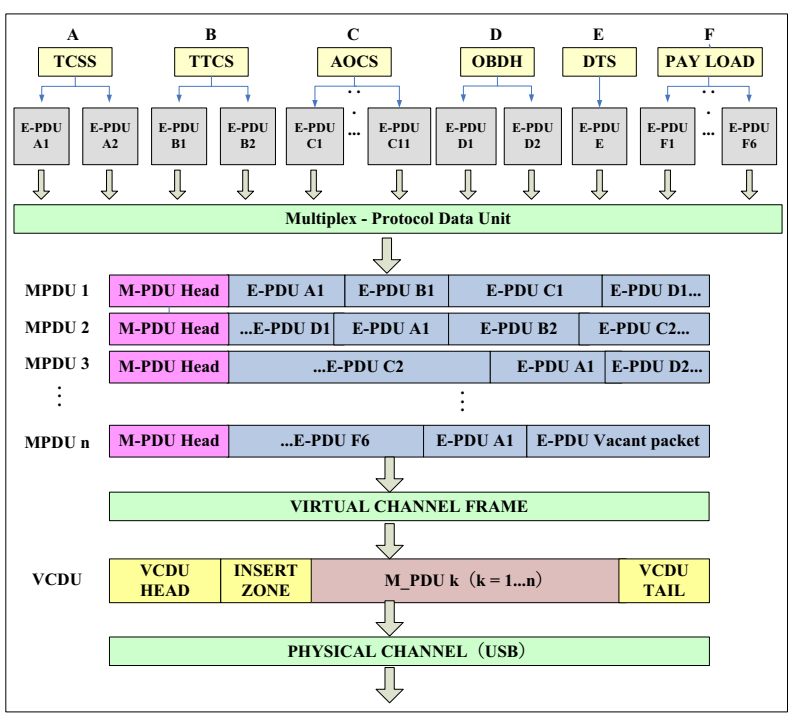

Fig. 4. TM data transmitting based on AOS

On-board time reference and timing signal are generated and distributed to satellite subsystems in-need. The OBDH S/S has the ability of generating the onboard time reference and distributing timing signals to the spacecraft subsystems. The on-board time reference shall be updated periodically according to the GPS clock or ground clock reference. The ways of concentrating time calibration and uniform time calibration should also be provided. Time reference is transferred to remote terminals via serial data bus There are three ways to correct the on board time, they are GPS time selfcalibration mode, GPS time coercive calibration for one time mode and TC calibration mode (concentrated time calibration and uniform time correction).

In addition, OBDH $\mathrm{S} / \mathrm{S}$ also provides the capability of housekeeping processing including system configuration, status data and event report collection, fault diagnosis, isolation and processing. Especially, $\mathrm{OBDH} \mathrm{S} / \mathrm{S}$ provides the capability of auto-control onboard, user-dedicated processing and data formatting. The serial data bus communication management is carried out by the OBDH S/S. Housekeeping functions of the CTU include system configuration, self-diagnosis and fault handling, telemetry scheduling mode switching, acquiring channel reconfiguration, and execution of the uplink control data, as well as the collection of the status data and historic event reports. Moreover, the CTU provides the functions of auto-control onboard, and userdedicated data generation, processing and distribution. The CTU is specifically designed to prevent the memory and components from SEU and SEL, and to provide the capability of memory write-protection.

\section{Flights software}

The OBDH F/S plays a relevant role in the implementation of the following functionality: management of the satellite AOCS, thermal control, spacecraft health management (also referred to as Failure Detection, Isolation, and Recovery (FDIR)), onboard autonomy, and spacecraft mode management. The OBDH F/S is composed of the CTU software, the RTU software, DRP software and TCU software. The CTU software is defined as management software, the RTU software is defined as execution software, DRP software is defined as data processing software and TCU software is defined as uplink data processing software.

The software of the CTU is the most important part in the OBDH, which is based on real-time operating system (RTOS). There are two hierarchical layers: one is nucleus which provides routines to serve all interfaces, and the other is application processes that implement the CTU tasks necessary. The CTU software is loaded in the PROM and run in the RAM.

The RTU software provides the functions of communication with the CTU through the SDB, telemetry acquisition (digital, analog and serial), command generation (on/off and serial) and internal OBDH processing.

The software architecture of the RTU, DRP \& TCU are sequential, which are loaded and executed in the PROM.

\subsection{Software Framework}

The development of the on board software should follow the software engineering standards and consider carefully the various aspects, for example, development methodology, quality assurance, configuration management and analysis/design methodology and tools. Furthermore, the capability should be provided by the flight software to support the unit testing of the OBDH $\mathrm{S} / \mathrm{S}$. 
During the process of development of data management sub-system on traditional spacecrafts, the management of protocol data form and data transaction take plenty time, because of the meticulous analysis of all kinds of transport protocols. A new design of spacecraft OBDH software framework based on onboard route is introduced in this article (see Fig.5). Using this framework, data link layer protocols, such as AosLink, TcLink and 1553Blink, are handled by network layer route, which works as a component. When application layer software needs to send/receive data, the sending/receiving interfaces of the network layer are utilized, and the background running route will choose the destination and service type needed for the data automatically, according to the route table. The data flow between ground, OBDH and other on board sub-systems is thus formed. This framework separates functions of the application layer from commutation on the bottom layer totally, which makes the application layer software design a lot more convenient [6-8].

\begin{tabular}{|c|c|c|c|c|c|}
\hline \begin{tabular}{|c|} 
Application \\
Layer
\end{tabular} & $\begin{array}{l}\text { Routine } \\
\text { Process }\end{array}$ & TM Process & TC Process & $\cdots$ & \begin{tabular}{|c|} 
Thermal \\
Control Process
\end{tabular} \\
\hline \multirow{5}{*}{$\begin{array}{c}\text { Component } \\
\text { Layer }\end{array}$} & TCLink & AosLink & BLib & DataPool & ThermoResistor \\
\hline & SeqQueue & QuickSort & Bus1553BLink & SynAynSelector & ThermoChannel \\
\hline & Record & IOBuffer & EleSelAccPeriod & DllLib & $\mathrm{TeVe}$ \\
\hline & IsoChecksum & CRC16 & Bits2Bytes & SpacePacket & TcMc \\
\hline & Byte2Bit & $\begin{array}{l}\text { TransBytesHi } \\
\text { gh2Low }\end{array}$ & $\begin{array}{c}\text { TransBytesLow2 } \\
\text { High }\end{array}$ & \multicolumn{2}{|l|}{$\cdots$} \\
\hline \multirow{2}{*}{$\begin{array}{c}\text { OS \& Driver } \\
\text { Layer }\end{array}$} & DeviceTcRecv & DeviceTmOut & 1553BDriver & $\cdots$ & DeviceOBTime \\
\hline & \multicolumn{5}{|c|}{ OS } \\
\hline $\begin{array}{c}\text { Physical } \\
\text { Layer }\end{array}$ & \multicolumn{5}{|c|}{ U、RAM、ROM、FPGA } \\
\hline
\end{tabular}

Fig. 5. OBDH software framework based on onboard route

\subsection{Software Maintenance}

The software maintenance in orbit is to fix and update CTU software through the uplink TC block to correct, replace some wrong software module, or to find a new application in order to satisfy new user requirements. Memory dump, program load, and process creation/activation/inactivation functions should be provided by the flight software maintenance in orbit.

\subsection{Software Tolerance}

\subsubsection{Important Data Store/Restore}

The important data should be able to store and to restore between the OBDH S/S and other subsystems. OBDH important data presents the OBDH operational state, interval of uniform correction; flag of sending safety switch-off command group, thermal auto-control, temperature range, payload initialize data, main/redundant table, antenna bias data, etc. Its purpose is that the previous working state makes a comeback when CTU resetting or switching.

$\mathrm{OBDH}$ important data shall be stored respectively in AOCC, RTU, and DRP while it updates. The OBDH important data and on-board time should be firstly restored from AOCC or RTUs, DRP to perform setup, CTU should also require AOCC to transmit AOCS important data to store when CTU power-on, resetting or switching.

\subsubsection{Software Auto-control}

There are several cases which could be able to cause CTU resetting by software (namely soft-reset), for example, Non-masked interrupt (watch-dog), RAM write-protect, RAM Hamming code checkout error, Process overtime and etc.

Under the permission of ground station, the CTU may be auto-switched by software normally, when the soft-reset occurs some times, or the CTU fails to communicate with all RTs.

The CTU unconditional auto-switch caused by software may occur at the instance of the failing of Memory self-test when CTU powered on, the wrong testing of TM bit rate, and the stop of On-board time.

\subsubsection{Data Estimation}

Some important TM parameters, for example, which participate in the controlling of satellite-launcher separation signal, should be judged at least three times continuously. If the testing results are identical, OBDH can make sure some event occurred. Considering the security of satellite, all data acquired by the RTU channels for satellite-launcher separation signal should be judged.

\subsubsection{Self-Testing and Self-Diagnosis}

OBDH software should provide the capability of selftesting and diagnosis for some critical interface such as $\mathrm{TM}$ video interface, and clock interface etc depending on the hardware. Error Detection and Correction Circuit (EDAC) should be adopted to correct the single-bit error and to detect the double-bit error for the memory. The memory should be refreshed periodically by using "read" operation to reduce the probability of piling up the single-bit error and to prevent from double-bit error, which has been proved to be an effective measure to prevent Signal Event Upsets (SEU).

\section{Redundancy and fault processing}

The following redundancies shall be provided by the OBDH subsystem. The TCU and CDU are dual redundant devices of "hot backup". The CTU, DRP and USO are designed to be dual redundant with "cold backup" spare unit. The serial data bus SDB is dual redundant and the second bus is in "hot backup" status. The routed TC redundancy shall be provided using two interconnected RTUs. The RTU is not required to be redundant for TMs. TM channels redundancy is implemented by routing TM to two RTUs. 
The OBDH S/S provides several fault tolerance techniques for failure protection, isolation and recovery. The criterion to determine the position when fault occurred is that, TM value used for testing, comparing or judging must be made a decision during a specified time limit. If the diagnosis result is "true" for three times continuously, the fault is determined to be happen.

In order to judge the fault occurrence, the OBDH S/S provides the capability of self-testing and self-diagnosis including CPU, RAM, PROM, analogue channel, serial digital channel, ON/OFF command channel, Memory Load command channel, TM/TC interfaces and on-board time, etc. Also, it provides the capability of collecting and recording the working status and event reports of the satellite.

Incorrect-write access protection memory, watch dog timer and the code selection filled in spare area of the memory are designed to prevent the program from deadloop and going astray. The OBDH S/S provides the capability of detection of error telecommand frame and important data, detection of application running overtime, and auto-selecting of telemetry data acquired by main and backup channels onboard, which gives the first priority to ground station to fill in the TM frame.

\section{Conclusions}

This paper has described the design of OBDH Subsystem for the China-Brazil Earth Resource Satellite based on onboard route. As described, the OBDH is composed by different units in order to provide data handling service to all the equipment of the satellite. The main units and functionalities of $\mathrm{OBDH}$ have been presented. An innovative flight software framework based on onboard route has been reported. In particular, attention has been paid to software maintenance and software tolerance. Finally, this paper has stressed the redundancy design and fault processing means provided by OBDH for ensuring the correctness.

\section{Acknowledgements}

This work has been supported by Project of China-Brazil Earth Resource Satellite.

\section{References}

1. Tan Weichi, Gu Yingqi . Space data system[M] . Beijing : China Science and Technology Press, 2004 (in Chinese).

2. Ralf Wenker, Cedric Legendre, etc. On-Board Software Architecture in MTG Satellite. The 4th IEEE International Workshop on Metrology for AeroSpace. 2017.

3. WANG Xianghui, WANG Tonghuan, LI Ningning, et al. An Efficient Scheduling Algorithm of Multiplexing TM Service Based on the AOS [J]. Beijing: SPACECRAFT ENGINEERING, 2011(in Chinese).
4. CCSDS, AOS Data Link Protocol. Recommendation for space data system standards, CCSDS 732.0-B-2. Blue Book[S]. Issue 1. Washington D.C.: CCSDS, 2006.

5. Space Packet Protocol. Recommendation for Space Data System Standards, CCSDS 133.0-B-1. Blue Book. Issue 1. Washington, D.C.: CCSDS, July 2003.

6. Garlan, D., Shaw, M. An introduction to software architecture. Technique Report, CMU/SEI-94-TR21, Carnegie Mellon University, 1994.

7. ZHANG Yahang, YUAN Jun, GUO Jian. Design of Reconfigurable Genral TM Based on Software Component [J]. Beijing: SPACECRAFT ENGINEERING, 2013(in Chinese).

8. ZHANG Yahang, ZHANG Meng, YuanJun, et al. Research of Spacecraft Software Components Based On C Language. The 1st Conference on High Graphics Earth Observation Technology. 2012.12(in Chinese). 